\title{
A Hankel matrix acting on Hardy and Bergman spaces
}

\author{
by \\ Petros Galanopoulos and José Ángel Peláez (Málaga)
}

Abstract. Let $\mu$ be a finite positive Borel measure on [0,1). Let $\mathcal{H}_{\mu}=\left(\mu_{n, k}\right)_{n, k \geq 0}$ be the Hankel matrix with entries $\mu_{n, k}=\int_{[0,1)} t^{n+k} d \mu(t)$. The matrix $\mathcal{H}_{\mu}$ induces formally an operator on the space of all analytic functions in the unit disc by the fomula

$$
\mathcal{H}_{\mu}(f)(z)=\sum_{n=0}^{\infty}\left(\sum_{k=0}^{\infty} \mu_{n, k} a_{k}\right) z^{n}, \quad z \in \mathbb{D},
$$

where $f(z)=\sum_{n=0}^{\infty} a_{n} z^{n}$ is an analytic function in $\mathbb{D}$.

We characterize those positive Borel measures on $[0,1)$ such that $\mathcal{H}_{\mu}(f)(z)=$ $\int_{[0,1)} \frac{f(t)}{1-t z} d \mu(t)$ for all $f$ in the Hardy space $H^{1}$, and among them we describe those for which $\mathcal{H}_{\mu}$ is bounded and compact on $H^{1}$. We also study the analogous problem for the Bergman space $A^{2}$.

1. Introduction. We denote by $\mathbb{D}=\{z \in \mathbb{C}:|z|<1\}$ the unit disc and by $\mathbb{T}$ the unit circle. Let $\mathcal{H}$ ol $(\mathbb{D})$ be the space of analytic functions in $\mathbb{D}$ and let $H^{p}(0<p \leq \infty)$ be the classical Hardy space of analytic functions in $\mathbb{D}$ (see $[\mathrm{D}]$ ).

If $0<p<\infty$ the Bergman space $A^{p}$ is the set of all $f \in \mathcal{H o l}(\mathbb{D})$ such that

$$
\|f\|_{A^{p}}^{p}:=\int_{\mathbb{D}}|f(z)|^{p} d A(z)<\infty,
$$

where $d A(z)=\pi^{-1} d x d y$ is the normalized Lebesgue area measure on $\mathbb{D}$. For the theory of these spaces we refer to [DS] and [Zh].

Let $\mu$ be a finite positive Borel measure on $[0,1)$ and let $\mathcal{H}_{\mu}=\left(\mu_{n, k}\right)_{n, k \geq 0}$ be the Hankel matrix with entries $\mu_{n, k}=\int_{[0,1)} t^{n+k} d \mu(t)$. The matrix $\mathcal{H}_{\mu}$ induces formally an operator (which will also be denoted $\mathcal{H}_{\mu}$ ) on $\mathcal{H o l}(\mathbb{D})$ in the following sense. If $f(z)=\sum_{n \geq 0} a_{n} z^{n} \in \mathcal{H} o l(\mathbb{D})$, by multiplication of the 
matrix with the sequence of Taylor coefficients of the function,

$$
\left\{a_{n}\right\}_{n \geq 0} \mapsto\left\{\sum_{k \geq 0} \mu_{n, k} a_{k}\right\}_{n \geq 0},
$$

we can formally define

$$
\mathcal{H}_{\mu}(f)(z)=\sum_{n=0}^{\infty}\left(\sum_{k=0}^{\infty} \mu_{n, k} a_{k}\right) z^{n}, \quad z \in \mathbb{D} .
$$

If $\mu$ is the Lebesgue measure on the interval $[0,1)$ we get the classical Hilbert matrix $\mathcal{H}=\left\{\frac{1}{n+k+1}\right\}_{n, k \geq 0}$. This matrix induces, in the same way as above, a bounded operator on $H^{p}, p \in(1, \infty)$ (see [DiS]), and on $A^{p}$, $p \in(2, \infty)$ (see $[\mathrm{Di}$ ); estimates on the norms have also been obtained. Recently in [DJV], a further progress has been achieved in this direction.

In this paper we shall focus our attention on the limit cases $H^{1}$ and $A^{2}$, that is, we shall study the boundedness, compactness, and other related properties of $\mathcal{H}_{\mu}$ on these spaces in terms of $\mu$. Similar investigations have previously been conducted by several authors in different spaces of analytic functions in $\mathbb{D}$ (see e.g. [W], [Po]).

The classical Hilbert matrix $\mathcal{H}$ is well defined but it is not bounded on $H^{1}$ (see [DiS]). It is known that the operator induced by the Hilbert matrix is not even well defined on $A^{2}$. Indeed, $f(z)=\sum_{n=1}^{\infty} \frac{1}{\log (n+1)} z^{n} \in A^{2}$ but $H f(0)=\sum_{n=1}^{\infty} \frac{1}{(n+1) \log (n+1)}=\infty$ (see [DJV]). Thus, it is natural to study under which conditions on the measure $\mu$ the corresponding matrix $\mathcal{H}_{\mu}$ induces a well defined and bounded operator on $H^{1}$ and on $A^{2}$.

The structure of the paper is as follows. In Section 2 we deal with the case of the Hardy space $H^{1}$. Let $\mu$ be a positive Borel measure in $\mathbb{D}$. For $\alpha \geq 0$ and $s>0$, we say that $\mu$ is an $\alpha$-logarithmic s-Carleson measure, resp. a vanishing $\alpha$-logarithmic s-Carleson measure, if

$$
\sup _{a \in \mathbb{D}} \frac{\mu(S(a))\left(\log \frac{2}{1-|a|^{2}}\right)^{\alpha}}{\left(1-|a|^{2}\right)^{s}}<\infty, \quad \text { resp. } \lim _{|a| \rightarrow 1^{-}} \frac{\mu(S(a))\left(\log \frac{2}{1-|a|^{2}}\right)^{\alpha}}{\left(1-|a|^{2}\right)^{s}}=0 .
$$

By $S(a)$ we denote the Carleson box with vertex at $a$, that is,

$$
S(a)=\left\{z \in \mathbb{D}: 1-|z| \leq 1-|a|,\left|\frac{\arg (a \bar{z})}{2 \pi}\right| \leq \frac{1-|a|}{2}\right\} .
$$

The above definition is a generalization of the fundamental notion of classical Carleson measure introduced by Carleson (see [C]). These are measures that occur for $\alpha=0$ and $s=1$.

We shall prove that any classical Carleson measure induces a well defined operator on $H^{1}$, and conversely being Carleson is necessary in the following sense. 
Proposition 1.1. Suppose that $\mu$ is a finite positive Borel measure on $[0,1)$.

(i) If $\mu$ is a classical Carleson measure then the power series $\mathcal{H}_{\mu}(f)(z)$ represents a function in $\mathcal{H o l}(\mathbb{D})$ for any $f \in H^{1}$, and moreover

$$
\mathcal{H}_{\mu}(f)(z)=\int_{[0,1)} \frac{f(t)}{1-t z} d \mu(t), \quad f \in H^{1} .
$$

(ii) If the integral in 1.2 converges for each $z \in \mathbb{D}$ and $f \in H^{1}$, then $\mu$ is a classical Carleson measure.

The hope that any classical Carleson measure $\mu$ induces a bounded operator $\mathcal{H}_{\mu}$ on $H^{1}$ is unjustified, because the Lebesgue measure does not. The next result describes the appropriate subclass of classical Carleson measures.

Theorem 1.2. Suppose that $\mu$ is a classical Carleson measure on $[0,1)$.

(i) $\mathcal{H}_{\mu}: H^{1} \rightarrow H^{1}$ is bounded if and only if $\mu$ is a 1-logarithmic 1Carleson measure.

(ii) $\mathcal{H}_{\mu}: H^{1} \rightarrow H^{1}$ is compact if and only if $\mu$ is a vanishing 1logarithmic 1-Carleson measure.

In many papers (see [CS], [JPS], [T], [PV] and [Pe]), another approach to the study of Hankel operators on spaces of analytic functions is developed, using the symbol of the operator, which in our case is essentially the function

$$
h_{\mu}(z)=\sum_{n} \mu_{n} z^{n}, \quad \mu_{n}=\int_{[0,1)} t^{n} d \mu(t) .
$$

A characterization of the boundedness and compactness of the operator $\mathcal{H}_{\mu}: H^{1} \rightarrow H^{1}$ in terms of $h_{\mu}$ follows from [PV, Theorems 1.6 and 1.7] (see also [CS], [JPS] and [T]). We shall provide two proofs of Theorem 1.2 , a first one based on the integral representation $(1.2)$ and a second one which uses the last cited result.

In the case of $H^{2}, \mathcal{H}_{\mu}$ is bounded if and only if $\mu$ is a classical Carleson measure (see [Pe]). Power, [Po, p. 428], proved that if $\int_{[0,1)} d \mu(t) /(1-t)^{2}<\infty$, then $\mathcal{H}_{\mu}$ is a Hilbert-Schmidt operator, and raised the question of a necessary condition. The next result solves this problem.

THEOREM 1.3. Let $\mu$ be a finite positive Borel measure on $[0,1)$ and suppose that the operator $\mathcal{H}_{\mu}$ is bounded on $H^{2}$. Then $\mathcal{H}_{\mu}$ is a HilbertSchmidt operator on $H^{2}$ if and only if

$$
\int_{[0,1)} \frac{\mu([t, 1))}{(1-t)^{2}} d \mu(t)<\infty .
$$


In Section 3 we turn our attention to $A^{2}$. First we clarify for which measures the operator is well defined on this space and also gets an integral representation.

Proposition 1.4. Let $\mu$ be a finite positive Borel measure on $[0,1)$.

(i) If $\mu$ satisfies (1.4) then the power series $\mathcal{H}_{\mu}(f)(z)$ is in $\mathcal{H o l}(\mathbb{D})$ for any $f \in A^{2}$ and moreover

$$
\mathcal{H}_{\mu}(f)(z)=\int_{[0,1)} \frac{f(t)}{1-t z} d \mu(t), \quad f \in A^{2} .
$$

(ii) If for any choice of $f \in A^{2}$ and $z \in \mathbb{D}$ the integral in 1.5 converges, then (1.4) is satisfied.

Unfortunately, condition (1.4) does not imply the boundedness of $\mathcal{H}_{\mu}$ on $A^{2}$ (see Theorem 1.5 and Proposition 1.7 below), so we need to look for a stronger one. Observe that (1.4) can be restated by saying that the analytic function $h_{\mu}$ belongs to the Dirichlet space

$$
\mathcal{D}=\left\{f(z)=\sum_{n=0}^{\infty} a_{n} z^{n} \in \mathcal{H} \text { ol }(\mathbb{D}): \int_{\mathbb{D}}\left|f^{\prime}(z)\right|^{2} d A(z)<\infty\right\},
$$

which is a Hilbert space equipped with the inner product $\langle f, g\rangle_{\mathcal{D}}=a_{0} \bar{b}_{0}+$ $\sum_{n \geq 0}(n+1) a_{n+1} \bar{b}_{n+1}$. We characterize in these terms the boundedness of the operator $\mathcal{H}_{\mu}$ on $A^{2}$.

THEOREM 1.5. Let $\mu$ be a finite positive Borel measure on $[0,1)$ that satisfies (1.4). The operator $\mathcal{H}_{\mu}$ is bounded in $A^{2}$ if and only if the measure $\left|h_{\mu}^{\prime}(z)\right|^{2} d A(z)$ is a Dirichlet Carleson measure.

We remind the reader that a finite positive Borel measure $\nu$ in $\mathbb{D}$ is called a Dirichlet Carleson measure if the identity operator is bounded from the Dirichlet space to $L^{2}(\mathbb{D}, \nu)$. We refer to [S] and [ARS] for descriptions of these measures.

It would be nice to relate the boundedness of the operator directly to a condition on the measure. In this spirit, we are able to describe the HilbertSchmidt operators on $A^{2}$.

Theorem 1.6. Let $\mu$ be a finite positive Borel measure on $[0,1)$ that satisfies (1.4). The operator $\mathcal{H}_{\mu}$ is a Hilbert-Schmidt operator on $A^{2}$ if and only if

$$
\int_{[0,1)} \frac{\mu([t, 1))}{(1-t)^{2}} \log \frac{1}{1-t} d \mu(t)<\infty
$$

Obviously, (1.6) gives bounded operators $\mathcal{H}_{\mu}$ on $A^{2}$; maybe surprisingly, it is sharp for the boundedness in a certain sense. 
Proposition 1.7. For each $\beta \in[0,1)$ there is a finite positive Borel measure $\mu$ on $[0,1)$ such that

$$
\int_{[0,1)} \frac{\mu([t, 1))}{(1-t)^{2}}\left(\log \frac{1}{1-t}\right)^{\beta} d \mu(t)<\infty,
$$

and $\mathcal{H}_{\mu}$ is not bounded on $A^{2}$.

2. The Hankel matrix $\mathcal{H}_{\mu}$ acting on $H^{1}$. Before we proceed to the proofs of Proposition 1.1 and Theorem 1.2 some results and definitions must be recalled. First, we present an equivalent description of the $\alpha$-logarithmic $s$-Carleson measures (see [Z]).

Lemma A. Suppose that $0 \leq \alpha<\infty$ and $0<s<\infty$ and $\mu$ is a positive Borel measure in $\mathbb{D}$. Then $\mu$ is an $\alpha$-logarithmic s-Carleson measure if and only if

$$
\sup _{a \in \mathbb{D}}\left(\log \frac{2}{1-|a|^{2}}\right)^{\alpha} \int_{\mathbb{D}}\left(\frac{1-|a|^{2}}{|1-\bar{a} z|^{2}}\right)^{s} d \mu(z)<\infty .
$$

We shall write $\mathrm{BMOA}_{\log , \alpha}, \alpha \geq 0$, (see [Gi] and [PV]) for the space of those $H^{1}$ functions whose boundary values satisfy

$$
\begin{aligned}
\|f\|_{\mathrm{BMOA}_{\log , \alpha}} & =|f(0)| \\
& +\sup _{a \in \mathbb{D}}\left(\log \frac{2}{1-|a|}\right)^{\alpha} \frac{1}{2 \pi} \int_{0}^{2 \pi}\left|f\left(e^{i \theta}\right)-f(a)\right| P_{a}\left(e^{i \theta}\right) d \theta<\infty,
\end{aligned}
$$

where $P_{a}\left(e^{i \theta}\right)=\left(1-|a|^{2}\right) /\left|1-a e^{-i \theta}\right|^{2}$ is the Poisson kernel.

We shall write $\mathrm{VMOA}_{\log , \alpha}$ for the subspace of $H^{1}$ of those functions $f$ such that

$$
\lim _{|a| \rightarrow 1^{-}}\left(\log \frac{2}{1-|a|}\right)^{\alpha} \int_{\mathbb{T}}\left|f\left(e^{i \theta}\right)-f(a)\right| P_{a}\left(e^{i \theta}\right) d \theta=0 .
$$

If $\alpha=0$, we obtain the classical space BMOA [VMOA] of $H^{1}$-functions with bounded [vanishing] mean oscillation. For simplicity, we shall write $\mathrm{BMOA}_{\log }\left[\mathrm{VMOA}_{\log }\right]$ for the space BMOA $A_{\log , 1}\left[\mathrm{VMOA}_{\log , 1}\right]$.

We shall also use Fefferman's result (see [Gi]) that $\left(H^{1}\right)^{*} \cong$ BMOA and $(\mathrm{VMOA})^{*} \cong H^{1}$, under the Cauchy pairing

$$
\begin{aligned}
\langle f, g\rangle_{H^{2}}=\lim _{r \rightarrow 1^{-}} \frac{1}{2 \pi} \int_{0}^{2 \pi} f\left(r e^{i \theta}\right) \overline{g\left(e^{i \theta}\right)} d \theta, \\
\quad f \in H^{1}, g \in \text { BMOA (resp. VMOA). }
\end{aligned}
$$

Proof of Proposition 1.1. (i) Let $f(z)=\sum_{n \geq 0} a_{n} z^{n} \in H^{1}$ and assume that $\mu$ is a classical Carleson measure. This means equivalently that (see 
[Pe, p. 42]) $\sup _{n \in \mathbb{N}} \mu_{n}(n+1)<\infty$. This fact together with Hardy's inequality (see [D, p. 48]) implies that

$$
\sum_{k=0}^{\infty} \mu_{n, k}\left|a_{k}\right| \leq C \sum_{k=0}^{\infty} \frac{\left|a_{k}\right|}{n+k+1} \leq C\|f\|_{H^{1}}, \quad n \in \mathbb{N},
$$

so $\mathcal{H}_{\mu}(f)(z) \in \mathcal{H o l}(\mathbb{D})$. The above inequalities also justify that

Then

$$
\sum_{k \geq 0} \mu_{n, k} a_{k}=\int_{[0,1)} t^{n} f(t) d \mu(t), \quad n \in \mathbb{N} .
$$

$$
\mathcal{H}_{\mu}(f)(z)=\sum_{n \geq 0}\left(\int_{[0,1)} t^{n} f(t) d \mu(t)\right) z^{n}=\int_{[0,1)} \frac{f(t)}{1-t z} d \mu(t), \quad z \in \mathbb{D} .
$$

The last equality is true since $\mu$ is a classical Carleson measure and so

$$
\sum_{n \geq 0}\left(\int_{[0,1)} t^{n}|f(t)| d \mu(t)\right)|z|^{n} \leq C\|f\|_{H^{1}} \frac{1}{1-|z|} .
$$

(ii) Assume that for any choice of $f \in H^{1}$ and $z \in \mathbb{D}$ the integral 1.2 converges. Fix $f \in H^{1}$ and choose $z=0$. This means that $\int_{[0,1)}|f(t)| d \mu(t)$ $<\infty$. If for any $\beta \in[0,1)$ we define $T_{\beta}: H^{1} \rightarrow L^{1}(d \mu)$ by setting $T_{\beta}(f)=$ $f \cdot \chi_{\{0 \leq|z|<\beta\}}$, then there is $C>0$ such that

$$
\left\|T_{\beta}(f)\right\|_{L^{1}(d \mu)}=\int_{[0, \beta)}|f(t)| d \mu(t) \leq \int_{[0,1)}|f(t)| d \mu(t) \leq C
$$

for any $\beta \in[0,1)$, which together with the uniform boundedness principle gives $\sup _{\beta \in[0,1)}\left\|T_{\beta}\right\|_{L^{1}(d \mu)}<\infty$, that is, the identity operator from $H^{1}$ to $L^{1}(d \mu)$ is bounded, thus by Carleson's result (see [D, Theorem 9.3]) $\mu$ is a classical Carleson measure.

Now we are ready to prove our main result in this section.

Proof of Theorem 1.2.

Proof of (i): Boundedness. We observe that the duality relation $(\mathrm{VMOA})^{*} \cong H^{1}$, Proposition 1.1. Cauchy's integral representation for functions in $H^{1}$ (see [D, Theorem 3.9]) and Fubini's theorem imply that

$$
\begin{aligned}
\mathcal{H}_{\mu}: & H^{1} \rightarrow H^{1} \text { is bounded } \\
\Leftrightarrow & \lim _{r \rightarrow 1^{-}}\left|\frac{1}{2 \pi} \int_{0}^{2 \pi}\left(\int_{0}^{1} \frac{f(t)}{1-t r e^{i \theta}} d \mu(t)\right) \overline{g\left(e^{i \theta}\right)} d \theta\right| \leq C\|f\|_{H^{1}}\|g\|_{\mathrm{BMOA}} \\
\Leftrightarrow & \lim _{r \rightarrow 1^{-}}\left|\int_{0}^{1} f(t) \overline{g(r t)} d \mu(t)\right| \leq C\|f\|_{H^{1}}\|g\|_{\mathrm{BMOA}}, \\
& \quad \text { for all } f \in H^{1} \text { and } g \in \mathrm{VMOA} .
\end{aligned}
$$


Suppose that $\mathcal{H}_{\mu}: H^{1} \rightarrow H^{1}$ is bounded and select the families of test functions

$$
g_{a}(z)=\log \frac{2}{1-a z}, \quad f_{b}(z)=\frac{1-b^{2}}{(1-b z)^{2}}, \quad a, b \in[0,1) .
$$

A calculation shows that $\left\{g_{a}\right\} \subset \mathrm{VMOA}$ and $\left\{f_{b}\right\} \subset H^{1}$ with

$$
\sup _{a \in[0,1)}\left\|g_{a}\right\|_{\mathrm{BMOA}}<\infty \quad \text { and } \sup _{b \in[0,1)}\left\|f_{b}\right\|_{H^{1}}<\infty .
$$

Next, taking $a=b \in[0,1)$ and $r \in[a, 1)$ we obtain

$$
\begin{aligned}
\left|\int_{0}^{1} f_{a}(t) \overline{g_{a}(r t)} d \mu(t)\right| & \geq \int_{a}^{1} \frac{1-a^{2}}{(1-a t)^{2}} \log \frac{2}{1-r a t} d \mu(t), \\
& \geq C \frac{\log \frac{2}{1-a^{2}}}{1-a^{2}} \mu([a, 1)),
\end{aligned}
$$

which bearing in mind (2.4) and (2.6) implies that $\mu$ is a 1-logarithmic 1-Carleson measure.

Conversely, suppose that $\mu$ is a 1-logarithmic 1-Carleson measure. Then by Lemma A,

$$
K_{\mu}:=\sup _{a \in \mathbb{D}} \log \frac{2}{1-|a|^{2}} \int_{\mathbb{D}} \frac{1-|a|^{2}}{|1-\bar{a} z|^{2}} d \mu(z)<\infty .
$$

Let us see that $\mathcal{H}_{\mu}$ is bounded on $H^{1}$. Using (2.4), it is enough to prove

$$
\begin{aligned}
& \lim _{r \rightarrow 1^{-}} \int_{0}^{1}|f(t)||g(r t)| d \mu(t) \leq C\|f\|_{H^{1}}\|g\|_{\mathrm{BMOA}} \\
& \qquad \text { for all } f \in H^{1} \text { and } g \in \mathrm{VMOA},
\end{aligned}
$$

which together with $[\mathrm{D}$, Theorem 9.3] and Lemma A is equivalent to

(2.9) $\quad \lim _{r \rightarrow 1^{-}} \sup _{a \in \mathbb{D}} \int_{\mathbb{D}} \frac{1-|a|^{2}}{|1-\bar{a} z|^{2}}|g(r z)| d \mu(z) \leq C\|g\|_{\mathrm{BMOA}} \quad$ for all $g \in$ VMOA.

On the other hand, for each $r \in(0,1), a \in \mathbb{D}$ and $g \in \mathrm{VMOA}$,

$$
\begin{aligned}
& \int_{\mathbb{D}} \frac{1-|a|^{2}}{|1-\bar{a} z|^{2}}|g(r z)| d \mu(z) \\
& \quad \leq|g(r a)| \int_{\mathbb{D}} \frac{1-|a|^{2}}{|1-\bar{a} z|^{2}} d \mu(z)+\int_{\mathbb{D}} \frac{1-|a|^{2}}{|1-\bar{a} z|^{2}}|g(r z)-g(r a)| d \mu(z) \\
& \quad=I_{1}(r, a)+I_{2}(r, a) .
\end{aligned}
$$


Bearing in mind that any function $g$ in the Bloch space $\mathcal{B}$ (see $\mathrm{ACP}$ ) has the growth

$$
|g(z)| \leq 2\|g\|_{\mathcal{B}} \log \frac{2}{1-|z|} \quad \text { for all } z \in \mathbb{D}
$$

and BMOA $\subset \mathcal{B}$ (see Theorem 5.1 of [Gi]), by (2.7) we have

$$
\begin{aligned}
I_{1}(r, a) & \leq C\|g\|_{\mathrm{BMOA}} \log \frac{2}{1-|a|} \int_{\mathbb{D}} \frac{1-|a|^{2}}{|1-\bar{a} z|^{2}} d \mu(z) \\
& \leq C K_{\mu}\|g\|_{\mathrm{BMOA}}<\infty \quad \text { for all } r \in(0,1) \text { and } a \in \mathbb{D} .
\end{aligned}
$$

Next, combining (2.7), [D, Theorem 9.3], 2.2) and the fact that BMOA is closed under subordination (see [Gi, Theorem 10.3]), we deduce that

$$
\begin{aligned}
I_{2}(r, a) & \leq C K_{\mu} \int_{\mathbb{T}} \frac{1-|a|^{2}}{\left|1-\bar{a} e^{i \theta}\right|^{2}}\left|g\left(r e^{i \theta}\right)-g(r a)\right| d \theta \\
& \leq C K_{\mu}\left\|g_{r}\right\|_{\mathrm{BMOA}} \\
& \leq C K_{\mu}\|g\|_{\mathrm{BMOA}} \quad \text { for all } r \in(0,1), a \in \mathbb{D} \text { and } g \in \mathrm{VMOA},
\end{aligned}
$$

which together with (2.10) and (2.11) implies (2.9).

Proof of (ii): Compactness. Suppose that $\mathcal{H}_{\mu}: H^{1} \rightarrow H^{1}$ is compact. Let $\left\{f_{b}\right\}$ be the family of functions defined in 2.5 and let $\left\{b_{n}\right\}$ be a sequence of points of $(0,1)$ such that $\lim _{n \rightarrow \infty} b_{n}=1$. Since $\left\{f_{b_{n}}\right\}$ is a bounded sequence in $H^{1}$, there is a subsequence $\left\{b_{n_{k}}\right\}$ and $g \in H^{1}$ such that $\lim _{k \rightarrow \infty}\left\|\mathcal{H}_{\mu}\left(f_{b_{n_{k}}}\right)-g\right\|_{H^{1}}=0$. Now, as $\left\{f_{b_{n_{k}}}\right\}$ converges to 0 uniformly on compact subsets of $\mathbb{D}$ and $\mu$ is a 1-logarithmic 1-Carleson measure, $\left\{\mathcal{H}_{\mu}\left(f_{b_{n_{k}}}\right)\right\}$ converges to 0 uniformly on compact subsets of $\mathbb{D}$, which implies that $g=0$. Thus, combining the fact that $\lim _{k \rightarrow \infty}\left\|\mathcal{H}_{\mu}\left(f_{b_{n_{k}}}\right)\right\|_{H^{1}}=0$ with the inequality (for all $g \in \mathrm{VMOA}$ )

$$
\lim _{r \rightarrow 1^{-}}\left|\int_{0}^{1} f_{b_{n_{k}}}(t) \overline{g(r t)} d \mu(t)\right| \leq C\left\|\mathcal{H}_{\mu}\left(f_{b_{n_{k}}}\right)\right\|_{H^{1}}\|g\|_{\mathrm{BMOA}},
$$

and the reasoning used in the boundedness case, we deduce that

$$
\lim _{k \rightarrow \infty} \frac{\mu\left(\left[b_{n_{k}}, 1\right)\right) \log \frac{2}{1-b_{n_{k}}}}{1-b_{n_{k}}}=0 .
$$

Consequently, $\mu$ is a vanishing 1-logarithmic 1-Carleson measure.

Conversely, assume that $\mu$ is a vanishing 1-logarithmic 1-Carleson measure. The proof of the sufficiency for the boundedness yields

$$
\int_{0}^{1}|f(t)||g(t)| d \mu(t) \leq C K_{\mu}\|f\|_{H^{1}}\|g\|_{\mathrm{BMOA}}
$$


So, it suffices to prove that for any sequence $\left\{f_{n}\right\}$ such that $\sup _{n \in \mathbb{N}}\left\|f_{n}\right\|_{H^{1}}$ $<\infty$ and $\lim _{n \rightarrow \infty} f_{n}=0$ on compact subsets of $\mathbb{D}$,

$$
\lim _{n \rightarrow \infty} \int_{0}^{1}\left|f_{n}(t)\right||g(t)| d \mu(t)=0 \quad \text { for all } g \in \text { VMOA. }
$$

Let us write $d \mu_{r}=\chi_{\{r<|z|<1\}} d \mu$. Since $\mu$ is a vanishing 1-logarithmic 1-Carleson measure, $\lim _{r \rightarrow 1^{-}} K_{\mu_{r}}=0$. This together with the fact that $\lim _{n \rightarrow \infty} f_{n}=0$ on compact subsets of $\mathbb{D}$, and (2.12), shows (using a standard argument) that $\mathcal{H}_{\mu}$ is compact on $H^{1}$.

In order to present a second proof of Theorem 1.2 some definitions and known results are needed. Given $g(\xi) \sim \sum_{n=-\infty}^{\infty} \hat{g}(n) \xi^{n} \in L^{2}(\mathbb{T})$, the associated Hankel operator (see $[\mathrm{Pe}]$ or $[\mathrm{PV}]$ ) is formally defined as

$$
H_{g}(f)=P(g J f)
$$

where $P$ is the Riesz projection and

$$
J f(\xi)=\bar{\xi} f(\bar{\xi})=\sum_{n=-\infty}^{\infty} \hat{f}(-n-1) \xi^{n}, \quad \xi \in \mathbb{T} .
$$

Moreover, if $\mu$ is a classical Carleson measure, Nehari's Theorem implies that (see $[\mathrm{Pe}, \mathrm{p} .3]$ or $\left[\mathrm{D}\right.$, Theorem 6.8]) there is $g_{\mu} \in L^{\infty}(\mathbb{T})$ with $\mu_{n}=$ $\hat{g}_{\mu}(n+1)$, so

$$
\mathcal{H}_{\mu}(f)(z)=\overline{H_{g_{\mu}}(f)(\bar{z})}
$$

and consequently $\mathcal{H}_{\mu}$ is bounded on $H^{1}$ if and only if $H_{g_{\mu}}$ is bounded on $H^{1}$. On the other hand,

$$
\begin{aligned}
P_{1}\left(g_{\mu}\right)(z) & :=P\left(g_{\mu}\right)(z)-\hat{g}_{\mu}(0)=\sum_{n=1}^{\infty} \hat{g}_{\mu}(n) z^{n}=\sum_{n=0}^{\infty} \hat{g}_{\mu}(n+1) z^{n+1} \\
& =\sum_{n=0}^{\infty} \mu_{n} z^{n+1}=z h_{\mu}(z) .
\end{aligned}
$$

Thus, we have the next result joining [PV, Theorems 1.6 and 1.7] (see also [CS], [JPS] and [T]).

Theorem A. Suppose that $\mu$ is a classical Carleson measure on $[0,1)$.

(i) $\mathcal{H}_{\mu}: H^{1} \rightarrow H^{1}$ is bounded if and only if $h_{\mu} \in \mathrm{BMOA}_{\log }$.

(ii) $\mathcal{H}_{\mu}: H^{1} \rightarrow H^{1}$ is compact if and only if $h_{\mu} \in \mathrm{VMOA}_{\log }$.

\section{Second proof of Theorem 1.2}

Proof of (i): Boundedness. If $\mathcal{H}_{\mu}: H^{1} \rightarrow H^{1}$ is bounded, then by Theorem A the function $h_{\mu}$ is in $\mathrm{BMOA}_{\log }$. For any $a \in(0,1)$ we deduce that 


$$
\begin{aligned}
\frac{1}{2 \pi} \int_{0}^{2 \pi}\left|h_{\mu}\left(e^{i \theta}\right)-h_{\mu}(a)\right| \frac{1-a^{2}}{\left|1-a e^{i \theta}\right|^{2}} d \theta \\
\quad=\frac{1}{2 \pi} \int_{0}^{2 \pi} \frac{1-a^{2}}{\left|1-a e^{i \theta \mid}\right|} \int_{0}^{1} \frac{t d \mu(t)}{\left(1-t e^{i \theta}\right)(1-t a)} \mid d \theta \\
\quad \geq \frac{1}{2 \pi} \int_{0}^{2 \pi} \frac{1-a^{2}}{\mid 1-a e^{i \theta \mid}} \operatorname{Re}\left(\int_{0}^{1} \frac{t d \mu(t)}{\left(1-t e^{i \theta}\right)(1-t a)}\right) d \theta \\
=\frac{1}{2 \pi} \int_{0}^{2 \pi} \frac{1-a^{2}}{\left|1-a e^{i \theta}\right|} \int_{0}^{1} \frac{t(1-t \cos (\theta))}{\left|1-t e^{i \theta}\right|^{2}(1-t a)} d \mu(t) d \theta \\
=\int_{0}^{1} \frac{t\left(1-a^{2}\right)}{1-t a}\left(\frac{1}{2 \pi} \int_{0}^{2 \pi} \frac{1-t \cos (\theta)}{\left|1-t e^{i \theta}\right|^{2} \mid 1-a e^{i \theta \mid}} d \theta\right) d \mu(t) \\
\geq \frac{1}{2} \int_{0}^{1} \frac{t\left(1-a^{2}\right)^{2}}{1-t a}\left(\frac{1}{2 \pi} \int_{0}^{2 \pi} \frac{1-t \cos (\theta)}{\left|1-t e^{i \theta}\right|^{2}\left|1-a e^{i \theta}\right|^{2}} d \theta\right) d \mu(t) .
\end{aligned}
$$

Assume, for the moment, that

$$
\frac{1}{2 \pi} \int_{0}^{2 \pi} \frac{1-t \cos (\theta)}{\left|1-t e^{i \theta}\right|^{2}\left|1-a e^{i \theta}\right|^{2}} d \theta=\frac{1}{(1-a t)\left(1-a^{2}\right)}
$$

for any $a, t \in[0,1)$.

This together with $(2.14)$ yields

$$
\sup _{a \in[0,1)} \log \frac{2}{1-a} \int_{0}^{1} \frac{t\left(1-a^{2}\right)}{(1-t a)^{2}} d \mu(t) \leq C\left\|h_{\mu}\right\|_{\mathrm{BMOA}_{\log }}<\infty,
$$

so $\mu$ is a 1 -logarithmic 1-Carleson measure.

Now, 2.15 will be proved. We assume that $a \neq t$ (if $a=t$ a similar calculation also gives (2.15) $)$, and we write

$$
F(z)=\frac{z-\frac{t}{2}\left(z^{2}+1\right)}{(z-t)(1-t z)(z-a)(1-a z)} .
$$

Therefore, using the residue theorem we see that

$$
\begin{aligned}
\frac{1}{2 \pi} \int_{0}^{2 \pi} \frac{1-t \cos (\theta)}{\left|1-t e^{i \theta}\right|^{2}\left|1-a e^{i \theta}\right|^{2}} d \theta & =\operatorname{Res}(F, t)+\operatorname{Res}(F, a) \\
& =\frac{\frac{t}{2}}{(t-a)(1-a t)}-\frac{a-\frac{t}{2}\left(a^{2}+1\right)}{(t-a)(1-a t)\left(1-a^{2}\right)} \\
& =\frac{1}{(1-a t)\left(1-a^{2}\right)},
\end{aligned}
$$

which proves (2.15). 
Conversely, suppose that $\mu$ is a 1-logarithmic 1-Carleson measure. Then $h_{\mu}$ has finite radial limit a.e. on $\mathbb{T}$, indeed $h_{\mu} \in H^{2}$ (see [Pe, p. 42]), and for any $a \in \mathbb{D}$,

$$
\begin{aligned}
& \frac{1}{2 \pi} \int_{0}^{2 \pi}\left|h_{\mu}\left(e^{i \theta}\right)-h_{\mu}(a)\right| \frac{1-|a|^{2}}{\mid 1-a e^{\left.i \theta\right|^{2}}} d \theta \\
& \quad=\frac{1}{2 \pi} \int_{0}^{2 \pi} \frac{1-|a|^{2}}{\left|1-a e^{i \theta \mid}\right|} \int_{0}^{1} \frac{t d \mu(t)}{\left(1-t e^{i \theta}\right)(1-t a)} \mid d \theta \\
& \leq \frac{1}{2 \pi} \int_{0}^{2 \pi} \frac{1-|a|^{2}}{\mid 1-a e^{i \theta \mid}} \int_{0}^{1} \frac{d \mu(t)}{\mid 1-t e^{i \theta|| 1-t a \mid} d \theta} \\
& \leq \frac{1-|a|^{2}}{2 \pi} \int_{0}^{1} \frac{1}{|1-t a|} \int_{0}^{2 \pi} \frac{d \theta}{\mid 1-a e^{i \theta|| 1-t e^{i \theta \mid}} d \mu(t)} \\
& \leq \frac{1-|a|^{2}}{2 \pi} \int_{0}^{1} \frac{1}{|1-t a|}\left(\int_{0}^{2 \pi} \frac{d \theta}{\left|1-a e^{i \theta}\right|^{2}}\right)^{1 / 2}\left(\int_{0}^{2 \pi} \frac{d \theta}{\left|1-t e^{i \theta}\right|^{2}}\right)^{1 / 2} d \mu(t) \\
& \leq C\left(1-|a|^{2}\right)^{1 / 2} \int_{0}^{1} \frac{1}{|1-t a|(1-t)^{1 / 2}} d \mu(t) \\
& \leq C\left(1-|a|^{2}\right)^{1 / 2} \int_{0}^{1} \frac{1}{(1-t|a|)(1-t)^{1 / 2}} d \mu(t) .
\end{aligned}
$$

Moreover, using that $\mu$ is a 1-logarithmic 1-Carleson measure and a standard argument (see [G] or [Z]) we conclude that

$$
\sup _{a \in(0,1)}\left(1-a^{2}\right)^{1 / 2} \int_{0}^{1} \frac{1}{(1-t a)(1-t)^{1 / 2}} d \mu(t)<\infty,
$$

which together with (2.16) shows that $h_{\mu} \in \mathrm{BMOA}_{\log }$, thus by Theorem A, $\mathcal{H}_{\mu}: H^{1} \rightarrow H^{1}$ is bounded.

The proof of (ii) is analogous, so it will be omitted.

Proof of Theorem 1.3. We recall that $\mathcal{H}_{\mu}$ is a Hilbert-Schmidt operator on $H^{2}$ if and only if $\sum_{k \geq 0}\left\|H_{\mu}\left(e_{k}\right)\right\|_{H^{2}}^{2}<\infty$ for any orthonormal base $\left\{e_{k}\right\}_{k=0}^{\infty}$. We choose the orthonormal base $e_{k}(z)=z^{k}$. For $z=r e^{i \theta} \in \mathbb{D}$, we observe that $\int_{0}^{2 \pi}\left|\mathcal{H}_{\mu}\left(e_{k}\right)\left(r e^{i \theta}\right)\right|^{2} d \theta=\sum_{n \geq 0}\left|\mu_{n, k}\right|^{2} r^{2 n}$. So

$$
\begin{aligned}
\sum_{k \geq 0}\left\|\mathcal{H}_{\mu}\left(e_{k}\right)\right\|_{H^{2}}^{2} & =\sum_{k \geq 0} \sum_{n \geq 0}\left|\mu_{n, k}\right|^{2}=\sum_{k \geq 0} \sum_{n \geq 0} \int_{[0,1)} \int_{[0,1)}(t s)^{n+k} d \mu(s) d \mu(t) \\
& =\int_{[0,1)} \int_{[0,1)} \frac{1}{(1-t s)^{2}} d \mu(s) d \mu(t) \approx \int_{[0,1)} \frac{\mu([t, 1))}{(1-t)^{2}} d \mu(t) .
\end{aligned}
$$

This finishes the proof. 
Finally, we shall see that although $\mathcal{H}_{\mu}$ is not bounded on $H^{1}$ for a classical Carleson measure $\mu$, in some sense $\mathcal{H}_{\mu}$ is close to having this property.

THEOREM 2.1. If $\mu$ is a classical Carleson measure supported on $[0,1)$ and $0<p<1$, then $\mathcal{H}_{\mu}: H^{1} \rightarrow H^{p}$ is bounded.

Proof. As $\mu$ is a classical Carleson measure,

$$
\begin{aligned}
& \left\|\mathcal{H}_{\mu}(f)\right\|_{H^{p}}^{p} \leq \sup _{0<r<1} \int_{-\pi}^{\pi}\left(\int_{[0,1)} \frac{|f(t)|}{\mid 1-t r e^{i \theta \mid}} d \mu(t)\right)^{p} d \theta \\
& \quad \leq C(\mu)\|f\|_{H^{1}}^{p} \sup _{0<r<1} \int_{-\pi}^{\pi} \sup _{0<t<1} \frac{1}{\left|1-t r e^{i \theta}\right|^{p}} d \theta \quad \text { for any } f \in H^{1} .
\end{aligned}
$$

On the other hand,

$$
\sup _{0<r<1} \sup _{0<t<1} \frac{1}{\left|1-t r e^{i \theta}\right|^{p}} \leq 1 \quad \text { if }|\theta| \geq \pi / 2,
$$

and a straightforward calculation shows that for $\theta \in(-\pi / 2, \pi / 2)$,

$$
\sup _{0<t<1} \frac{1}{\left|1-t r e^{i \theta}\right|^{p}} \leq \max \left\{\frac{1}{\left|1-r e^{i \theta}\right|^{p}}, \frac{1}{\sin ^{p}(\theta)}\right\},
$$

which together with 2.17 and 2.18 finishes the proof.

Indeed, the previous result must be improved. We remind the reader that $f \in \mathcal{H o l}(\mathbb{D})$ is a Cauchy transform if it admits a representation

$$
f(z)=\int_{0}^{2 \pi} \frac{d \nu(\theta)}{1-e^{i \theta} z}, \quad z \in \mathbb{D},
$$

where $\nu$ is a finite complex valued Borel measure on $\mathbb{T}$. As usual, $\mathcal{K}$ will denote the space of all Cauchy transforms. It is known (see [CSi]) that $\bigcap_{0<p<1} H^{p} \subsetneq \mathcal{K} \subsetneq H^{1}$ and moreover $\mathcal{K}$ is isometrically isomorphic (under the Cauchy pairing) to the dual space of $\mathcal{A}$, the disk algebra, which consists of all $g \in \mathcal{H o l}(\mathbb{D})$ such that $g$ is continuous on $\overline{\mathbb{D}}$. This allows us to assert that

$$
\|f\|_{\mathcal{K}}=\sup \left\{\langle f, g\rangle_{H^{2}}: g \in \mathcal{A},\|g\|_{H^{\infty}} \leq 1\right\} .
$$

THEOREM 2.2. If $\mu$ is a classical Carleson measure supported on $[0,1)$ then $\mathcal{H}_{\mu}: H^{1} \rightarrow \mathcal{K}$ is bounded.

Proof. Putting together the fact that $\mu$ is a classical Carleson measure, Proposition 1.1. Cauchy's integral representation for functions in $H^{1}$ and 
Fubini's theorem we deduce that for $f \in H^{1}$ and $g \in \mathcal{A}$,

$$
\begin{aligned}
\lim _{r \rightarrow 1^{-}} \mid \frac{1}{2 \pi} \int_{0}^{2 \pi}\left(\int_{0}^{1} \frac{f(t)}{1-t r e^{i \theta}}\right. & d \mu(t)) \overline{g\left(e^{i \theta}\right)} d \theta \mid \\
& =\lim _{r \rightarrow 1^{-}}\left|\int_{0}^{1} f(t) \overline{g(r t)} d \mu(t)\right| \\
& \leq\|g\|_{H^{\infty}} \int_{0}^{1}|f(t)| d \mu(t) \leq C\|f\|_{H^{1}}\|g\|_{H^{\infty}},
\end{aligned}
$$

so $\mathcal{H}_{\mu}: H^{1} \rightarrow \mathcal{K}$ is bounded.

In particular, Theorem 2.2 implies that for any $f \in H^{1}, \mathcal{H}_{\mu}(f)\left(e^{i \theta}\right)$ is finite for a.e. $e^{i \theta}$ on $\mathbb{T}$. Indeed, a little more can be said.

Proposition 2.3. If $\mu$ is a classical Carleson measure supported on $[0,1)$ then the operator $\mathcal{H}_{\mu}$ is of weak type $(1,1)$ on Hardy spaces. That is, there is a positive constant $C$ such that

$$
\left|\left\{e^{i \theta} \in \mathbb{T}:\left|\mathcal{H}_{\mu}(f)\left(e^{i \theta}\right)\right| \geq \lambda\right\}\right| \leq \frac{C}{\lambda}\|f\|_{H^{1}} \quad \text { for all } f \in H^{1} .
$$

Proof. Using that $\mu$ is a classical Carleson measure and Nehari's theorem (see $[\mathrm{Pe}, \mathrm{p} .3]$ or $\left[\mathrm{D}\right.$, Theorem 6.8]) we deduce that there is $g \in L^{\infty}(\mathbb{T})$ such that

$$
\mu_{n}=\frac{1}{2 \pi} \int_{0}^{2 \pi} e^{-i n t} g(t) d t=: \hat{g}(n), \quad n=0,1,2, \ldots
$$

Then, by [DJV, Theorem 1],

$$
\mathcal{H}_{\mu}(f)=P M_{g} T(f) \quad \text { for all } f \in \bigcup_{p>1} H^{p}
$$

where $T f\left(e^{i t}\right)=f\left(e^{-i t}\right)$ and $M_{g}$ is the multiplication operator by $g$. Thus, using standard techniques and well-known results we deduce that $\mathcal{H}_{\mu}$ is of weak type $(1,1)$ on Hardy spaces.

3. The Hankel matrix $\mathcal{H}_{\mu}$ acting on $A^{2}$. We recall that the Bergman projection $\operatorname{Pf}(z)=\int_{\mathbb{D}} f(w) \overline{K_{z}(w)} d A(w)$ is bounded from $L^{2}(d A)$ to $A^{2}$ (see [Zh]), where $K_{z}(w)=(1-\bar{z} w)^{-2}$ is the Bergman kernel of $A^{2}$. It follows that any $f \in A^{2}$ can be represented by its Bergman projection and moreover $\left(A^{2}\right)^{*} \cong A^{2}$ under the pairing $\langle f, g\rangle_{A^{2}}=\int_{\mathbb{D}} f(z) \overline{g(z)} d A(z)$.

Proof of Proposition 1.4. (i) Fix $n \in \mathbb{N}$. If $f(z)=\sum_{k=0}^{\infty} a_{k} z^{k} \in A^{2}$, then by the Cauchy-Schwarz inequality, 


$$
\left|\sum_{k \geq 0} \mu_{n, k} a_{k}\right| \leq \sum_{k \geq 0} \mu_{n, k}\left|a_{k}\right| \leq\left\{\sum_{k \geq 0}(k+1) \mu_{n, k}^{2}\right\}^{1 / 2}\|f\|_{A^{2}}
$$

But

$$
\begin{aligned}
\sum_{k \geq 0}(k+1) \mu_{n, k}^{2} & =\int_{[0,1)} \int_{[0,1)} \frac{(t s)^{n}}{(1-t s)^{2}} d \mu(s) d \mu(t) \\
& =2 \int_{[0,1)} \int_{[t, 1)} \frac{(t s)^{n}}{(1-t s)^{2}} d \mu(s) d \mu(t) \leq 2 \int_{[0,1)} \frac{\mu([t, 1))}{(1-t)^{2}} d \mu(t) .
\end{aligned}
$$

Thus, if $\mu$ satisfies (1.4) the power series (1.1) is well defined and it represents an analytic function in $\mathbb{D}$. Under (1.4) we can also write

$$
\sum_{k \geq 0} \mu_{n, k} a_{k}=\int_{[0,1)} t^{n} f(t) d \mu(t) .
$$

So, for $z \in \mathbb{D}$,

$$
\mathcal{H}_{\mu}(f)(z)=\sum_{n \geq 0}\left(\int_{[0,1)} t^{n} f(t) d \mu(t)\right) z^{n}=\int_{[0,1)} \frac{f(t)}{1-t z} d \mu(t) .
$$

The last equality is true since

$$
\sum_{n \geq 0}\left(\int_{[0,1)} t^{n}|f(t)| d \mu(t)\right)|z|^{n} \leq\left\{2 \int_{[0,1)} \frac{\mu([t, 1))}{(1-t)^{2}} d \mu(t)\right\}^{1 / 2}\|f\|_{A^{2}} \frac{1}{1-|z|} .
$$

(ii) Take $f \in A^{2}$. Assume that the integral in 1.5 converges for each $z \in \mathbb{D}$. We choose $z=0$. So, there is $C>0$ such that

$$
\left|\int_{[0, \beta)} f(t) d \mu(t)\right| \leq \int_{[0, \beta)}|f(t)| d \mu(t) \leq \int_{[0,1)}|f(t)| d \mu(t) \leq C
$$

for all $\beta \in(0,1)$.

On the other hand, the integral representation of $f \in A^{2}$ through the Bergman projection, and Fubini's theorem, imply that

$$
\begin{aligned}
\int_{[0, \beta)} f(t) d \mu(t) & =\int_{[0, \beta)} \int_{\mathbb{D}} \frac{f(w)}{(1-\bar{w} t)^{2}} d A(z) d \mu(t) \\
& =\int_{\mathbb{D}} f(w) \overline{\int_{[0, \beta)} \frac{1}{(1-w t)^{2}} d \mu(t)}=\left\langle f, g_{\beta}\right\rangle_{A^{2}},
\end{aligned}
$$

where $g_{\beta}(w)=\int_{[0, \beta)} \frac{1}{(1-w t)^{2}} d \mu(t) \in A^{2}$ for every $\beta$. Then, combining (3.3), the fact that $\left(A^{2}\right)^{*} \cong A^{2}$ under the pairing $\langle\cdot, \cdot\rangle_{A^{2}}$, and the uniform bound- 
edness principle, we conclude that $\sup _{\beta}\left\|g_{\beta}\right\|_{A^{2}}<C$. Thus, using that $\left\|g_{\beta}\right\|_{A^{2}}^{2}=\int_{[0, \beta)} \int_{[0, \beta)} \frac{1}{(1-t s)^{2}} d \mu(s) d \mu(t)$, we get

$$
C \geq \int_{[0,1)} \int_{[0,1)} \frac{1}{(1-t s)^{2}} d \mu(s) d \mu(t) \geq \frac{1}{4} \int_{[0,1)} \frac{\mu([t, 1))}{(1-t)^{2}} d \mu(t) .
$$

So condition (1.4) is true.

Proof of Theorem 1.5. It is known that $\left(A^{2}\right)^{*} \cong \mathcal{D}$ and $\mathcal{D}^{*} \cong A^{2}$ under the Cauchy pairing $\langle f, g\rangle_{H^{2}}=\sum_{n \geq 0} a_{n} \bar{b}_{n}$ where $f(z)=\sum_{n} a_{n} z^{n}$ $\in A^{2}$ and $g(z)=\sum_{n} b_{n} z^{n} \in \mathcal{D}$. We observe that, under this relation, $\mathcal{H}_{\mu}$ is self-adjoint. Therefore, $\mathcal{H}_{\mu}$ is bounded on $A^{2}$ if and only if it is on $\mathcal{D}$.

If $f, g \in \mathcal{D}$ we shall write $f_{1}(z)=\sum_{n}\left|a_{n}\right| z^{n}, g_{1}(z)=\sum_{n}\left|b_{n}\right| z^{n}$ so that $\|f\|_{\mathcal{D}}=\left\|f_{1}\right\|_{\mathcal{D}}$ and $\|g\|_{\mathcal{D}}=\left\|g_{1}\right\|_{\mathcal{D}}$. Then

$$
\begin{aligned}
\left|\left\langle\mathcal{H}_{\mu}(f), g\right\rangle_{\mathcal{D}}\right| & \\
\leq & \sum_{n \geq 0}(n+1)\left(\sum_{k \geq 0} \mu_{n+1, k}\left|a_{k}\right|\right)\left|b_{n+1}\right|+\mu_{0}\left|a_{0}\right|\left|b_{0}\right|+\left|b_{0}\right| \sum_{k=0}^{\infty} \mu_{k+1}\left|a_{k+1}\right| \\
\leq & \sum_{n \geq 0} \mu_{n+1}\left(\sum_{k=0}^{n}(k+1)\left|b_{k+1}\right|\left|a_{n-k}\right|\right)+\mu_{0}\|f\|_{\mathcal{D}}\|g\|_{\mathcal{D}} \\
& +\|g\|_{\mathcal{D}} \int_{\mathbb{D}}\left(\frac{f_{1}(z)-f_{1}(0)}{z}\right) \overline{h_{\mu}^{\prime}(z)} d A(z) \\
\leq & \int_{\mathbb{D}} f_{1}(z) g_{1}^{\prime}(z) \overline{h_{\mu}^{\prime}(z)} d A(z)+\mu_{0}\|f\|_{\mathcal{D}}\|g\|_{\mathcal{D}} \\
& \quad+\|g\|_{\mathcal{D}} \int_{\mathbb{D}}\left(\frac{f_{1}(z)-f_{1}(0)}{z}\right) \overline{h_{\mu}^{\prime}(z)} d A(z) .
\end{aligned}
$$

So, if $\left|h_{\mu}^{\prime}(z)\right|^{2} d A(z)$ is a Dirichlet Carleson measure, we get

$$
\begin{aligned}
\left|\left\langle\mathcal{H}_{\mu}(f), g\right\rangle_{\mathcal{D}}\right| & \\
& \leq\left\{\int_{\mathbb{D}}\left|f_{1}(z)\right|^{2}\left|h_{\mu}^{\prime}(z)\right|^{2} d A(z)\right\}^{1 / 2}\left\{\int_{\mathbb{D}}\left|g_{1}^{\prime}(z)\right|^{2} d A(z)\right\}^{1 / 2}+\mu_{0}\|f\|_{\mathcal{D}}\|g\|_{\mathcal{D}} \\
& +\left\{\int_{\mathbb{D}}\left|\frac{f_{1}(z)-f_{1}(0)}{z}\right|^{2}\left|h_{\mu}^{\prime}(z)\right|^{2} d A(z)\right\}^{1 / 2}\left\{\int_{\mathbb{D}}\left|g_{1}^{\prime}(z)\right|^{2} d A(z)\right\}^{1 / 2} \\
& \leq C\|f\|_{\mathcal{D}}\|g\|_{\mathcal{D}},
\end{aligned}
$$

and consequently $\mathcal{H}_{\mu}$ is bounded. 
Conversely, assume that $\mathcal{H}_{\mu}$ is bounded on $\mathcal{D}$. Then

$$
\begin{aligned}
\mid \int_{\mathbb{D}} f(z) g^{\prime}(z) \overline{h_{\mu}^{\prime}(z)} & d A(z) \mid \\
& \leq \int_{0}^{1} \sum_{n \geq 0}(n+1) \mu_{n+1}\left(\sum_{k=0}^{n}(k+1)\left|b_{k+1}\right|\left|a_{n-k}\right|\right) r^{n+1} d r \\
& \leq \sum_{n \geq 0}(n+1)\left(\sum_{k \geq 0} \mu_{n+1, k}\left|a_{k}\right|\right)\left|b_{n+1}\right| \\
& =\left|\left\langle\mathcal{H}_{\mu}\left(f_{1}\right), g_{1}\right\rangle_{\mathcal{D}}\right| \leq C\|f\|_{\mathcal{D}}\|g\|_{\mathcal{D}} .
\end{aligned}
$$

So (exchanging also the roles of $f$ and $g$ ) we have

$$
\left|\int_{\mathbb{D}}(f g)^{\prime}(z) \overline{h_{\mu}^{\prime}(z)} d A(z)\right| \leq C\|f\|_{\mathcal{D}}\|g\|_{\mathcal{D}}
$$

for every $f, g \in \mathcal{D}$. Finally, Theorem 1 of ARSW] (see also [Wu] implies that $\left|h_{\mu}^{\prime}(z)\right|^{2} d A(z)$ is a Dirichlet Carleson measure.

REMARK 3.1. We recall that ARS, Theorem 1] says that a positive Borel measure $\nu$ in $\mathbb{D}$ is a Dirichlet Carleson measure if and only if there is a positive constant $C$ such that for all $a \in \mathbb{D}$,

$$
\int_{\tilde{S}(a)}(\nu(S(z) \cap S(a)))^{2} \frac{d A(z)}{\left(1-|z|^{2}\right)^{2}} \leq C \nu(S(a)),
$$

where

$$
\tilde{S}(a)=\left\{z \in \mathbb{D}: 1-|z| \leq 2(1-|a|),\left|\frac{\arg (a \bar{z})}{2 \pi}\right| \leq \frac{1-|a|}{2}\right\} .
$$

We note that if $\nu$ is finite, (3.4) is equivalent to the simpler condition

$$
\int_{S(a)}(\nu(S(z) \cap S(a)))^{2} \frac{d A(z)}{\left(1-|z|^{2}\right)^{2}} \leq C \nu(S(a)),
$$

because in this case

$$
\begin{aligned}
\int_{\tilde{S}(a) \backslash S(a)}(\nu(S(z) & \cap S(a)))^{2} \frac{d A(z)}{\left(1-|z|^{2}\right)^{2}} \\
& \leq C(1-|a|)^{-2} \int_{\tilde{S}(a) \backslash S(a)}(\nu(S(z) \cap S(a)))^{2} d A(z) \\
& \leq C(1-|a|)^{-2} \nu(S(a))^{2} \int_{\tilde{S}(a) \backslash S(a)} d A(z) \leq C \nu(S(a)) .
\end{aligned}
$$

Consequently, combining Proposition 1.4 and Theorem 1.5, if $\mu$ is a finite positive Borel measure on $[0,1)$ that satisfies (1.4), $\mathcal{H}_{\mu}$ is bounded in $A^{2}$ if and only if the measure $\nu=\left|h_{\mu}^{\prime}(z)\right|^{2} d A(z)$ satisfies $(3.5)$ for all $a \in \mathbb{D}$. 
Proof of Theorem 1.6. Take the orthonormal basis $\left\{e_{k}\right\}_{k \geq 0}=(k+1)^{1 / 2} z^{k}$ and observe that

$$
\begin{aligned}
\sum_{k=0}^{\infty}\left\|\mathcal{H}_{\mu}\left(e_{k}\right)\right\|_{A^{2}}^{2} & =\sum_{k=0}^{\infty}(k+1) \sum_{n=0}^{\infty}(n+1)^{-1} \mu_{n, k}^{2} \\
& =\sum_{k=0}^{\infty}(k+1) \iint_{0}^{1}(t s)^{k} \frac{1}{t s} \log \frac{1}{1-t s} d \mu(t) d \mu(s) \\
& \asymp \int_{[0,1)} \frac{\mu([t, 1))}{(1-t)^{2}} \log \frac{1}{1-t} d \mu(t) .
\end{aligned}
$$

So the operator is Hilbert-Schmidt if and only if (1.6) holds.

Finally we shall prove Proposition 1.7 .

Proof of Proposition 1.7. We claim that if $\mathcal{H}_{\mu}$ is bounded on $A^{2}$ then

$$
\sup _{a \in(0,1)} \frac{\int_{[0,1)} \frac{\mu([t, 1))}{(1-t)^{2}}\left(\frac{1}{a t} \log \frac{1}{1-a t}\right)^{2} d \mu(t)}{\frac{1}{a^{2}} \log \frac{1}{1-a^{2}}}<\infty .
$$

Assume (3.7) for the moment. Let $\beta \in[0,1), \alpha \in((1+\beta) / 2,1)$ and consider the measure $d \mu_{\alpha}(t)=\left(\frac{1}{t} \log \frac{1}{1-t}\right)^{-\alpha} d t$. Using that $\mu_{\alpha}([t, 1)) \asymp$ $(1-t)\left(\frac{1}{t} \log \frac{1}{1-t}\right)^{-\alpha}$, we deduce

$$
\int_{0}^{1} \frac{\mu_{\alpha}([t, 1))}{(1-t)^{2}}\left(\frac{1}{t} \log \frac{1}{1-t}\right)^{\beta} d \mu_{\alpha}(t) \asymp \int_{0}^{1} \frac{1}{(1-t)}\left(\frac{1}{t} \log \frac{1}{1-t}\right)^{\beta-2 \alpha} d t<\infty
$$

and

$$
\begin{aligned}
& \left(\frac{1}{a^{2}} \log \frac{1}{1-a^{2}}\right)^{-1} \int_{[0,1)} \frac{\mu_{\alpha}([t, 1))}{(1-t)^{2}}\left(\frac{1}{a t} \log \frac{1}{1-a t}\right)^{2} d \mu_{\alpha}(t) \\
& \quad \geq C\left(\frac{1}{a^{2}} \log \frac{1}{1-a^{2}}\right)^{-1} \int_{[0, a)} \frac{1}{1-t}\left(\frac{1}{t} \log \frac{1}{1-t}\right)^{-2 \alpha}\left(\frac{1}{t^{2}} \log \frac{1}{1-t^{2}}\right)^{2} d t \\
& \quad \geq C\left(\log \frac{1}{1-a}\right)^{2-2 \alpha}
\end{aligned}
$$

which in particular implies that

$$
\lim _{a \rightarrow 1^{-}}\left(\frac{1}{a^{2}} \log \frac{1}{1-a^{2}}\right)^{-1} \int_{[0,1)} \frac{\mu_{\alpha}([t, 1))}{(1-t)^{2}}\left(\frac{1}{a t} \log \frac{1}{1-a t}\right)^{2} d \mu_{\alpha}(t)=\infty .
$$

So, $\mu_{\alpha}$ does not satisfy (3.7) and thus $\mathcal{H}_{\mu_{\alpha}}$ is not bounded. 
In order to prove $(3.7)$, using that $\left(A^{2}\right)^{*} \cong A^{2}$ under the pairing $\langle,\rangle_{A^{2}}$, we obtain

$$
\begin{aligned}
& \mathcal{H}_{\mu}: A^{2} \rightarrow A^{2} \text { is bounded } \\
\Leftrightarrow & \left|\int_{\mathbb{D}}\left(\int_{[0,1)} \frac{f(t)}{1-t z} d \mu(t)\right) \overline{g(z)} d A(z)\right| \leq C\|f\|_{A^{2}}\|g\|_{A^{2}} \text { for all } f, g \in A^{2} .
\end{aligned}
$$

Set $g_{a}(z)=\frac{1}{1-a z}, a \in(0,1)$. Then $\left\|g_{a}\right\|_{A^{2}}^{2}=\frac{1}{a^{2}} \log \frac{1}{1-a^{2}}$ and

$$
\begin{aligned}
\int_{\mathbb{D}} \frac{g_{a}(z)}{1-t \bar{z}} d A(z) & =\int_{\mathbb{D}}\left(\sum_{n=0}^{\infty}(a z)^{n}\right)\left(\sum_{n=0}^{\infty}(t \bar{z})^{n}\right) d A(z) \\
& =\frac{1}{a t} \log \frac{1}{1-a t}, \quad a, t \in(0,1) .
\end{aligned}
$$

Then, by (3.8) (with $g=g_{a}$ ) and Fubini's theorem, we get

$$
\sup _{a \in(0,1)}\left|\int_{0}^{1} f(t) d \mu_{a}(t)\right| \leq C\|f\|_{A^{2}} \quad \text { for all } f \in A^{2},
$$

where

$$
d \mu_{a}(t)=\frac{\frac{1}{a t} \log \frac{1}{1-a t}}{\left(\frac{1}{a^{2}} \log \frac{1}{1-a^{2}}\right)^{1 / 2}} d \mu(t) .
$$

So, there is $C>0$ such that

$$
\sup _{a, \beta \in(0,1)}\left|\int_{0}^{\beta} f(t) d \mu_{a}(t)\right| \leq C\|f\|_{A^{2}} \quad \text { for all } f \in A^{2} .
$$

Next, arguing as in the the proof of Proposition 1.4, we obtain

$$
\sup _{a, \beta \in(0,1)}\left\|\int_{0}^{\beta} \frac{d \mu_{a}(t)}{(1-w t)^{2}}\right\|_{A^{2}}<\infty,
$$

which together with the fact that

$$
\begin{aligned}
& \left\|\int_{0}^{\beta} \frac{d \mu_{a}(t)}{(1-w t)^{2}}\right\|_{A^{2}}^{2}=\sum_{n=0}^{\infty}(n+1)\left[\int_{0}^{\beta} t^{n} d \mu_{a}(t)\right]^{2} \\
& \geq\left(\frac{1}{a^{2}} \log \frac{1}{1-a^{2}}\right)^{-1} \sum_{n=0}^{\infty}(n+1) \int_{0}^{\beta} t^{2 n}\left(\frac{1}{a t} \log \frac{1}{1-a t}\right)^{2} \mu([t, \beta)) d \mu(t) \\
& \geq \frac{1}{4}\left(\frac{1}{a^{2}} \log \frac{1}{1-a^{2}}\right)^{-1} \int_{0}^{\beta} \frac{\left(\frac{1}{a t} \log \frac{1}{1-a t}\right)^{2}}{(1-t)^{2}} \mu([t, \beta)) d \mu(t)
\end{aligned}
$$

finishes the proof. 
Acknowledgements. The authors wish to thank Professor A. Aleman for his helpful comments and for interesting discussions on the topic of the paper.

The first author is partially supported by the European Networking Programme "HCAA" of the European Science Foundation. The second author is partially supported by the Ramón y Cajal program of MICINN (Spain). Both authors are supported by grants from "Ministerio de Educación y Ciencia, Spain" (MTM2007-60854) and from "La Junta de Andalucía" (FQM210) and P09-FQM-4468.

\section{References}

[ACP $]$ J. M. Anderson, J. Clunie and Ch. Pommerenke, On Bloch functions and normal functions, J. Reine Angew. Math. 270 (1974), 12-37.

[ARS] N. Arcozzi, R. Rochberg and E. Sawyer, Carleson measures for analytic Besov spaces, Rev. Mat. Iberoamer. 18 (2002), 443-510.

[ARSW] N. Arcozzi, R. Rochberg, E. Sawyer and B. Wick, Bilinear forms on the Dirichlet space, Anal. PDE 3 (2010), 21-47.

[C] L. Carleson, An interpolation problem for bounded analytic functions, Amer. J. Math. 80 (1958), 921-930.

[CS] J. Cima and D. Stegenga, Hankel operators on $H^{p}$, in: Analysis at Urbana. Vol. 1: Analysis in Function Spaces, London Math. Soc. Lecture Note Ser. 137, Cambridge Univ. Press, Cambridge, 1989), 133-150.

[CSi] J. Cima and A. Siskakis, Cauchy transforms and Cesàro averaging operators, Acta Sci. Math. (Szeged) (1999), 505-513.

[Di] E. Diamantopoulos, Hilbert matrix on Bergman spaces, Illinois J. Math. 48 (2004), 1067-1078.

[DiS] E. Diamantopoulos and A. Siskakis, Composition operators and the Hilbert matrix, Studia Math. 140 (2000), 191-198.

[DJV] M. Dostanić, M. Jevtić and D. Vukotić, Norm of the Hilbert matrix on Bergman and Hardy spaces and a theorem of Nehari type, J. Funct. Anal. 254 (2008), 2800-2815.

[D] P. L. Duren, Theory of $H^{p}$ Spaces, Academic Press, New York, 1970. Reprint: Dover, Mineola, NY, 2000.

[DS] P. L. Duren and A. P. Schuster, Bergman Spaces, Math. Surveys Monogr. 100, Amer. Math. Soc., Providence, RI, 2004.

[G] J. B. Garnett, Bounded Analytic Functions, Academic Press, 1981.

[Gi] D. Girela, Analytic functions of bounded mean oscillation, in: Complex Functions Spaces, R. Aulaskari (ed.), Univ. Joensuu Dept. Math. Rep. Ser. 4 (2001), 61-171.

[JPS] S. Janson, J. Petree and S. Semmes, On the action of Hankel and Toeplitz operators on some function spaces, Duke Math. J. 51 (1984), 937-958.

[PV] M. Papadimitrakis and J. A. Virtanen, Hankel and Toeplitz operators on $H^{1}$ : continuity, compactness and Fredholm properties, Integral Equations Operator Theory 61 (2008), 573-591.

[Pe] V. Peller, Hankel Operators and Their Applications, Springer Monogr. Math., Springer, New York, 2003. 
[Po] S. C. Power, Hankel operators on Hilbert space, Bull. London Math. Soc. 12 (1980), 422-442.

[S] D. Stegenga, Multipliers of the Dirichlet space, Illinois J. Math. 24 (1980), 113-139.

[T] V. A. Tolokonnikov, Hankel and Toeplitz operators in Hardy spaces, Zap. Nauchn. Sem. Leningrad. Otdel. Mat. Inst. Steklov. (LOMI) 141 (1985), 165-175 (in Russian); English transl.: J. Soviet Math. 37 (1987), 1359-1364.

[W] H. Widom, Hankel matrices, Trans. Amer. Math. Soc. 121 (1966), 1-35.

[Wu] Z. Wu, The dual and second predual of $W_{\sigma}$, J. Funct. Anal. 116 (1993), 314-334.

[Z] R. Zhao, On logarithmic Carleson measures, Acta Sci. Math. (Szeged) 69 (2003), 605-618.

[Zh] K. Zhu, Operator Theory in Function Spaces, I, II, 2nd ed., Cambridge Univ. Press, Cambridge, 1959.

Petros Galanopoulos, José Ángel Peláez

Departamento de Análisis Matemático

Universidad de Málaga

Campus de Teatinos, 29071 Málaga, Spain

E-mail: galanopoulos_petros@yahoo.gr

japelaez@uma.es

Received December 9, 2009

Revised version May 26, 2010

$(6764)$ 